\title{
A scenario for black hole evaporation on a quantum geometry
}

\section{Rodolfo Gambini}

Instituto de Física, Facultad de Ciencias, Montevideo, Uruguay

E-mail: rgambini@fisica.edu.uy

\section{Jorge Pullin*}

Department of Physics and Astronomy, Louisiana State University, Baton Rouge, LA 70803

E-mail: pullinalsu.edu

\begin{abstract}
We incorporate elements of the recently discovered exact solutions of the quantum constraints of loop quantum gravity for vacuum spherically symmetric space-times into the paradigm of black hole evaporation due to Ashtekar and Bojowald. The quantization of the area of the surfaces of symmetry of the solutions implies that the number of nice slices that can be fit inside the black hole is finite. The foliation eventually moves through the region where the singularity in the classical theory used to be and all the particles that fell into the black hole due to Hawking radiation emerge finally as a white hole. This yields a variant of a scenario advocated by Arkani-Hamed et al. Fluctuations in the horizon that naturally arise in the quantum space time allow radiation to emerge during the evaporation process due to stimulated emission allowing evaporation to proceed beyond Page time without reaching the maximum entanglement limit until the formation of the white hole. No firewalls nor remnants arise in this scenario.
\end{abstract}

Frontiers of Fundamental Physics 14 - FFP14,

15-18 July 2014

Aix Marseille University (AMU) Saint-Charles Campus, Marseille

\footnotetext{
* Speaker.
} 
In a recent development, the exact space of solutions of the equations of loop quantum gravity for vacuum, spherically symmetric space-times was found [1]. This was due to the realization that a rescaling and linear combination of the constraints of canonical quantum gravity in the spherical case yields a constraint algebra that is a Lie algebra. This allows to complete the Dirac quantization procedure for the model. Remarkably, the space of physical states can be found in closed form. It is based on one dimensional spin networks. The proximity of the nodes of the spin networks is bounded below by the condition of the quantization of the areas of the spheres of symmetry. The singularity that is inside black holes in classical general relativity is replaced by a region where a description in terms of a semiclassical geometry is not possible and through it one tunnels into another region of space-time. Although this solution corresponds to an eternal time independent black hole, elements from its description can be heuristically incorporated into black hole evaporation scenarios. Concretely, there are two lessons from the eternal black hole model that can be extended to the evaporating case: a) The number of nice slices that can be fit into the black hole interior is finite due to the quantization of areas present in the quantum space-time; b) the second lesson is that the Dirac observable associated with the position of the horizon (the ADM mass) undergoes fluctuations that grow with time. We will see these elements can have interesting implications for the resulting scenario.

A paradigm for black hole evaporation in loop quantum gravity was put forward by Ashtekar and Bojowald [3]. Motivated by loop quantum gravity analyses of the black hole interior treating it as a Kantowski-Sachs cosmology [4] and of results in CGHS 1+1 dimensional model [5], the picture that emerges is the one depicted in 1 . As a star collapses to form a black hole, a trapping horizon forms, initially space-like near $r=0$ and eventually becomes time-like, ending up by touching the region where a semiclassical space-time description is not available.

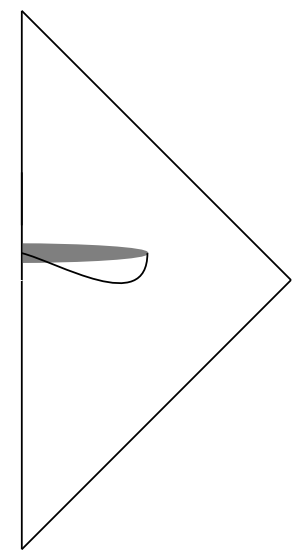

Figure 1: The Penrose diagram of the Ashtekar-Bojowald paradigm. As matter collapses, a trapping horizon forms, initially spatial and eventually timelike. The shaded region is not well approximated by a semiclassical space-time and is where the classical singularity was.

We would like to argue that when one incorporates elements of the new exact solution, namely, the spatial discreteness implied by the quantization of area in loop quantum gravity and the fact that states are superpositions with different masses and spin networks, to the above paradigm, a new picture emerges that introduces small but significant modifications to Susskind's notion of black hole complementarity [6]. The three principles of black hole complementarity are: 1) That the 
evolution is unitary, there is an S matrix describing the evolution of infalling matter to Hawking radiation. This is unmodified. 2) Outside the horizon, physics can be described by quantum field theory on a classical background with small but important quantum gravity corrections. In our case the background is represented by a geometry given by the expectation values of the metric on suitable quantum states that approximate the classical behavior. The quantum gravity effects imply fluctuations in the position of the horizon that play an important role in providing important departures from quantum field theory in curved space time at times of the order of the Page time. 3) To a distant observer a black hole appears as a quantum system with discrete energy levels for the purposes of entropy calculations. In our case, this is implied by the quantization of the areas, as we will note later. In addition it is usually assumed that an observer falling into the black hole does not notice anything unusual when crossing the horizon [7]. These points remain unchanged in our analysis.

A standard way to describe Hawking radiation is to consider the construction of "nice slices" $[8,9]$. These are spatial slices of the space-time that penetrate the horizon interpolating between $t=$ const. slices in the exterior to $r=$ const. slices in the interior (in Schwarzschild coordinates). This requires a stretching in the region around the horizon. The stretching implies that the vacuum on one slice is not the vacuum on future slices and this implies creation of pairs of particles and accounts for the Hawking radiation. Each particle emitted in the exterior becomes entangled with a particle inside the black hole. For an observer in the asymptotic region, the radiation appears thermal, since part of the information is lost, at least locally, and one needs to trace over the degrees of freedom not accessible behind the horizon. The slices are usually constructed with a spacing in the exterior of the black hole of the order of twice the Schwarzschild radius. This ensures that the radiation produced has the energy corresponding to the maximum of the standard spectrum of Hawking radiation.

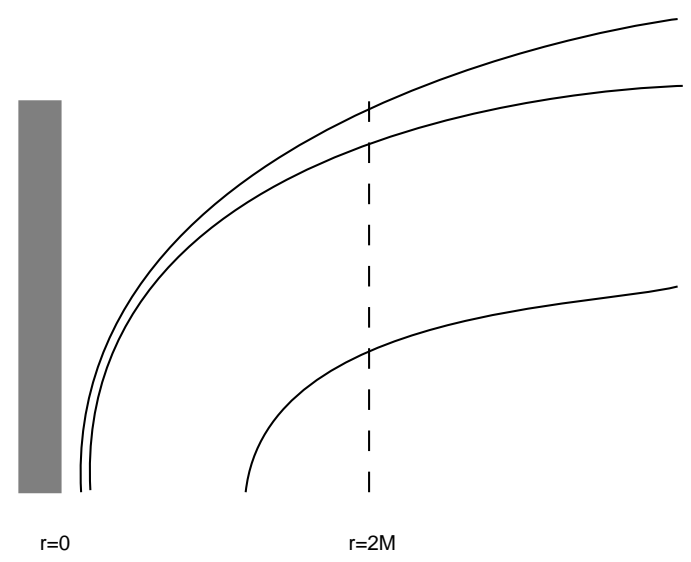

Figure 2: The "nice slices" that enter the black hole and whose stretching is responsible for the Hawking radiation. Outside they asymptote to surfaces $t=$ const. and inside to $r=$ const. in ordinary Schwarzschild coordinates.

One of the new elements introduced by the quantum space-time of loop quantum gravity is that the separation of the slices is bounded below by the rule of the quantization of the area of the spheres of spherical symmetry. The background quantum space-time that emerges from the new exact solution for the physical space of states in spherical symmetry is based on one dimensional 
spin networks. One has a graph $g$ on which the network is based and a set of integers $\vec{k}$ that are the valences the edges of the spin network. The values that the areas of the spheres of symmetry can take on these states are $4 \pi \ell_{\text {Planck }}^{2} k$ at the various links of the spin network. This implies that for the slices in the interior of the black hole that are close to the singularity one has a separation in the radial coordinate of order $\ell_{\text {Planck }}$. Elsewhere, the separation of the slices is bounded below by $\ell_{\text {Planck }}^{2} /(2 r)$. The value $k=0$ has to be eliminated in order to have a self-adjoint metric operator and that implies that the singularity present in the classical Schwarzschild solution is eliminated in this approach. This in turn means that in the interior of the black hole can only accommodate a finite number of slices bounded above by $\operatorname{Int}\left((2 G M)^{2} / \ell_{\text {Planck }}^{2}\right)$. Although we have based our discussion on a model of eternal black hole in loop quantum gravity, a similar description with the same Dirac observables is available in models that involve collapse, as can be illustrated with collapsing shells. In reference [10] we discuss the collapse of test shells and in a manuscript in preparation we extend the results to self-gravitating shells.

Remarkably, this simple one dimensional model of black hole reproduces correctly the entropy calculation. If one considers all possible states in the interior, its number is bounded by

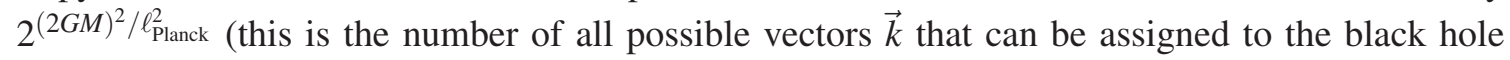
interior for a given mass $M$ ). Its logarithm then yields an entropy proportional to the area in Planck units with a factor of order unity.

In turn, this means that from the point of view of an observer far away from the black hole, the traditional evaporation process lasts a time $\operatorname{Int}\left((2 G M)^{2} / \ell_{\text {Planck }}^{2}\right)(4 G M) / c$. This gives $16 G^{3} M^{3} /\left(\hbar G c^{4}\right)$, which is of the order of magnitude of the Page time. At this time, the nice slices reach the region where the classical singularity used to be and quantum field theory in curved space-time is not a good approximation anymore to the relevant physics. Once the slices traverse the region where the singularity was in the classical theory, all the particles that fell in due to Hawking radiation during the evaporation emerge in a white hole. Notice that the previous evaporation time is only an order of magnitude estimate, we are ignoring several important effects, including the black hole evaporation in its calculation.

After the slices cross the region where the classical singularity used to be and become more and more like ordinary slices of Minkowski space, the usual Hawking evaporation switches off and the particles that fell into the black hole are allowed to emerge. At this point we cannot give a precise picture of how the radiation that emerges after the traditional evaporation stops behaves in detail. That would require studying how the particles that fell into the black hole (and are entangled with the earlier outgoing radiation) interact with the region of high curvature surrounding where the classical singularity used to be. A preliminary study of shells crossing the region, exhibiting some of the relevant effects can be seen in [10]. This work is currently being extended to self gravitating shells. Once the nice slices went through that region, they have it in their past and therefore even their discussion requires a full quantum treatment.

An objection that could be raised to this scenario is that as the black hole evaporates, it is well know that the interior becomes more and more entangled with the outgoing radiation until there are not enough degrees of freedom left in the interior to continue the evaporation [11]. Therefore Hawking evaporation should stop in a regime where quantum field theory in curved space time should work very well. This, among other reasons, led to the proposal of firewalls to avoid this problem [7]. It would also imply that the scenario we are proposing could not take place. This 
point of view does not take into account that in a situation with a quantum geometry, one will have fluctuations of the position of the horizon. In the exact solution presented in [1] this is taken into account since the mass of the black hole is a Dirac observable with continuous spectrum and the generic solution representing a classical space time will be given by a superposition of states with different values of the mass, centered in a given classical value. That means that the quantum state near the horizon will fluctuate from being a vacuum into having particles. Specifically, every time a particle of the Hawking radiation is emitted, the mass of the black hole will fluctuate by an amount given by the time-energy uncertainty relation. The characteristic time for emission is given by the separation of the "nice slices" and is therefore of the order of $2 G M / c$. The horizon therefore carries out a "random walk" in position and after a Page time the fluctuation in its position is of the order of the Planck mass. This implies a separation in the distance between the inner and outer extrema of the fluctuations of the order of $2 \int_{r_{S}}^{r_{S}+\ell_{\text {Planck }}}{\sqrt{1-r_{S} / r}}^{-1} d r=2 \sqrt{2 G M \ell_{\text {Planck }}}+O\left(\ell_{\text {Planck }}^{3 / 2}\right)$. So it is a cumulative effect on the position of the horizon due to the back-reaction of the particle production [12]. The large differences in characteristic frequencies associated with the positions of the horizon in the fluctuation — due to the high blueshifts close to the horizon—, imply that the vacuum in one position will be significantly different than the vacuum in the other. This would lead to stimulated emission in the Hawking radiation of similar nature to that due to thermal and other types of fluctuations of the horizon in black hole analogue systems [13]. A heuristic picture is that a particle created by pair production for a horizon will stimulate emission of particles with the same quantum numbers for a horizon further out as the position of the horizon fluctuates due to quantum effects. This extra emission allows the black hole to lose mass without increasing the entanglement entropy as the outgoing particle generated by stimulated emission is not entangled with any infalling particle. Stimulated emission (with a very different origin) has been proposed [14] as a solution of the information problem by itself, however it does not appear be a strong enough effect to account for the exit of all the information at the end of the process. Our scenario does not face this problem as the information can further exit in significant quantities through the white hole (plus the effect leading to stimulated emission appears larger). Hawking radiation will fail to be perfectly thermal due to stimulated emission. This will manifest itself in fluctuations in the temperature. The radiation may also exhibit a certain degree of coherence or non-thermality due to the stimulated emission, absent in ordinary Hawking radiation. In other words, entanglement grows at a smaller rate than the one predicted by ordinary Hawking radiation. These deviations could be calculated in detail and the magnitude of this effect estimated with the quantum spacetime techniques of [15] but they would require certain modifications of how one treats the matter Hamiltonian, that exceed the scope of this note. So at this point we conjecture that the combined effect of spontaneous and stimulated emission will allow the evaporation to continue till the white hole is formed but this remains to be confirmed.

This mechanism of finite number of nice slices therefore provides a variant of an implementation of a proposal by Arkani-Hamed et al. [9]. In their original proposal the limitation was given by bounds on the accuracy for measuring times between two successive slices that was heuristic. Here such limitation arises from the loop quantization of the space-time and the traditional evaporation switches off gradually after the slices make it through the region where the classical singularity used to be.

Since in our approach there is no singularity, the particles of the Hawking radiation that fell 


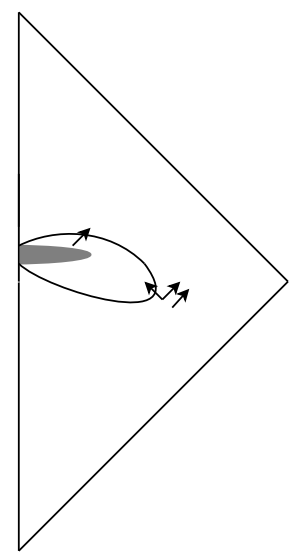

Figure 3: The proposed paradigm. A trapping horizon forms during the Hawking radiation period. When the radiation ends one has a white hole that radiates the information that had fallen into the trapping horizon in the process of generation of Hawking radiation and no remnant is left behind. The arrows indicate particles of the Hawking radiation outgoing and infalling into the black hole and particles of the white hole exiting.

into the black hole can in principle traverse the region where the classical singularity used to be. The detailed description of that traversing is involved, but in principle there is no reason for the information that fell into the black hole not to get out by traversing the region where the singularity used to be. The resulting picture is that of a white hole, with considerable similarity to the original white hole proposal of Hawking [16]: "To an external observer a white hole is indistinguishable from a black hole. The process of hole formation and evaporation is completely time-symmetric. The irreversibility which arises in the classical limit is just a statistical effect". In the case we are considering there is no reason for the process to be time-symmetric, though.

As the picture that emerges is that of a white hole, it suggests that no "remnant" is produced, although the true details will depend on the dynamics in the region of high curvature which at the moment we do not have. Asymptotically, the information could therefore potentially be retrieved. The information of the first particle of each produced pair could be retrieved at infinity through the usual Hawking radiation (and the stimulated emission) and the information of the particle that fell into the black hole is radiated to infinity in the white hole phase. What we are doing in our proposal is to start with a black hole that morphs into a white hole. However, since the particles that emerge through the white hole had complicated interactions in the region of high curvature, the radiation of the white hole is not the same as the one produced during the black hole phase. This has some similarities with the model considered by Parikh and Wilczek [17] in that there is a region of space-time that is in causal contact with a region where one needs the full quantum theory for its description and that is the source of the asymmetry in the radiation. In particular in the white hole phase, it is unlikely to have a purely thermal spectrum. Notice that some of the arguments given against white holes that argue they are unstable are based on the existence of Cauchy horizons for the space-time, which in the Ashtekar-Bojowald picture do not emerge.

Notice that we have respected complementarity, there was no need to invoke firewalls [7] and the information is naturally retrieved completely without remnants. However, although we have a scenario where quantum field theory in curved spacetime applies locally in the regions of low 
curvature, our construction requires slices that went through a region where there is not a classical space-time description which will modify usual predictions in the final stages of evaporation. In particular traditional Hawking radiation switches off and is replaced by emission of particles that had fallen into the black hole. An important caveat is that our calculations are based on spherical symmetry. It is reassuring that remarkably, the entropy calculation works out (at an orderof-magnitude level) and that the bound on the number of slices can be arrived at from different perspectives, that are also valid without spherical symmetry. Loop quantum gravity calculations of Hawking radiation are now available [15] and in particular imply the elimination of singularities in physical quantities, like the expectation value of the stress-energy tensor. This opens up the possibility of carrying out back reaction calculations. With this, it will be possible relatively soon to further explore the presented paradigm with detailed calculations.

We wish to thank Don Marolf for many helpful discussions. This work was supported in part by grant NSF-PHY-1305000, funds of the Hearne Institute for Theoretical Physics, CCT-LSU and Pedeciba.

\section{References}

[1] R. Gambini and J. Pullin, Phys. Rev. Lett. 110, no. 21, 211301 (2013) [arXiv:1302.5265 [gr-qc]]; R. Gambini, J. Olmedo and J. Pullin, Class. Quant. Grav. 31, 095009 (2014) [arXiv:1310.5996 [gr-qc]].

[2] R. Gambini and J. Pullin, “Quantum shells in a quantum space-time," arXiv:1408.4635 [gr-qc].

[3] A. Ashtekar and M. Bojowald, Class. Quant. Grav. 22, 3349 (2005) [gr-qc/0504029].

[4] L. Modesto, Int. J. Theor. Phys. 45, 2235 (2006) [gr-qc/0411032]; A. Ashtekar, M. Bojowald A. Ashtekar and M. Bojowald, Class. Quant. Grav. 23, 391 (2006) [gr-qc/0509075]; Böhmer and Vandersloot C. G. Boehmer and K. Vandersloot, Phys. Rev. D 76, 104030 (2007) [arXiv:0709.2129 [gr-qc]]; R. Gambini, J. Pullin, M. Campiglia, R. Gambini and J. Pullin, AIP Conf. Proc. 977, 52 (2008) [arXiv:0712.0817 [gr-qc]].

[5] Phys. Rev. Lett. 106, 161303 (2011) [arXiv:1011.6442 [gr-qc]]; Phys. Rev. D 83, 044040 (2011) [arXiv:1012.0077 [gr-qc]].

[6] L. Susskind, L. Thorlacius and J. Uglum, Phys. Rev. D 48, 3743 (1993) [hep-th/9306069].

[7] A. Almheiri, D. Marolf, J. Polchinski and J. Sully, JHEP 1302, 062 (2013) [arXiv:1207.3123 [hep-th]]; A. Almheiri, D. Marolf, J. Polchinski, D. Stanford and J. Sully, JHEP 1309, 018 (2013) [arXiv:1304.6483 [hep-th]]; see also S. L. Braunstein, [arXiv:0907.1190v1 [quant-ph]] published as S. L. Braunstein, S. Pirandola and K. Zyczkowski, Physical Review Letters 110, 101301 (2013) for a similar prediction from different assumptions.

[8] S. D. Mathur, Class. Quant. Grav. 26, 224001 (2009) [arXiv:0909.1038 [hep-th]].

[9] N. Arkani-Hamed, S. Dubovsky, A. Nicolis, E. Trincherini and G. Villadoro, JHEP 0705, 055 (2007) [arXiv:0704.1814 [hep-th]].

[10] R. Gambini, J. Pullin "Collapsing shells in a quantum space-time", in preparation.

[11] D. N. Page, JCAP 1309, 028 (2013) [arXiv:1301.4995 [hep-th]].

[12] B. L. Hu and A. Roura, Int. J. Theor. Phys. 46, 2204 (2007) [gr-qc/0601088]. 
[13] T. Jacobson, Lect. Notes Phys. 870, 1 (2013) [arXiv:1212.6821].

[14] C. Adami and G. L. Ver Steeg, Class. Quant. Grav. 31, 075015 (2014) [gr-qc/0407090].

[15] R. Gambini and J. Pullin, Class. Quant. Grav. 31, 115003 (2014) [arXiv:1312.3595 [gr-qc]].

[16] S. W. Hawking, Phys. Rev. D 13, 191 (1976).

[17] M. K. Parikh and F. Wilczek, Phys. Lett. B 449, 24 (1999) [gr-qc/9807031]. 